University of Nebraska - Lincoln

DigitalCommons@University of Nebraska - Lincoln

$1-2017$

\title{
PTSD, Alcohol Dependence, and Conduct Problems: Distinct Pathways via Lability and Disinhibition
}

Jeffrey S. Simons

Raluca M. Simons

Carol O'Brien

Scott F. Stoltenberg

Jessica A. Keith

See next page for additional authors

Follow this and additional works at: https://digitalcommons.unl.edu/psychfacpub

Part of the Genetics Commons, and the Psychology Commons

This Article is brought to you for free and open access by the Psychology, Department of at

DigitalCommons@University of Nebraska - Lincoln. It has been accepted for inclusion in Faculty Publications, Department of Psychology by an authorized administrator of DigitalCommons@University of Nebraska - Lincoln. 


\section{Authors}

Jeffrey S. Simons, Raluca M. Simons, Carol O'Brien, Scott F. Stoltenberg, Jessica A. Keith, and Jaime A. Hudson 


\title{
PTSD, Alcohol Dependence, and Conduct Problems: Distinct Pathways via Lability and Disinhibition
}

\author{
Jeffrey S. Simons, ${ }^{1,4}$ Raluca M. Simons, ${ }^{1,4}$ Carol O'Brien, ${ }^{2}$ \\ Scott F. Stoltenberg, ${ }^{3}$ Jessica A. Keith, ${ }^{2}$ and Jaime A. Hudson ${ }^{4}$
}

1. The University of South Dakota, Vermillion, South Dakota, USA

2. Bay Pines VA Health Care System, Bay Pines, Florida, USA

3. The University of Nebraska-Lincoln, Lincoln, Nebraska, USA

4. Sioux Falls VA Medical Center, Sioux Falls, South Dakota, USA

Corresponding author - Jeffrey S. Simons, University of South Dakota, Vermillion, email jeffrey.simons@usd.edu

\begin{abstract}
This study tested the role of affect lability and disinhibition in mediating associations between PTSD symptoms and two forms of alcohol-related problems, dependence syndrome symptoms (e.g., impaired control over consumption) and conduct problems (e.g., assault, risk behaviors). Genotype at the serotonin transporter linked polymorphic region (5-HTTLPR) was hypothesized to moderate associations between traumatic stress and PTSD symptoms. In addition, the study tested whether childhood traumatic stress moderated associations between combat trauma and PTSD symptoms. Participants were $270 \mathrm{OIF} / \mathrm{OEF} / \mathrm{OND}$ veterans. The hypothesized model was largely supported. Participants with the low expression alleles of 5-HTTLPR ( $\mathrm{S}$ or $\mathrm{LG}$ ) exhibited stronger associations between childhood (but not combat) traumatic stress and PTSD symptoms. Affect lability mediated the associations between PTSD symptoms and alcohol dependence symptoms. Behavioral disinhibition mediated associations between PTSD symptoms and conduct related problems. Conditional indirect effects indicated stronger associations between childhood traumatic stress and lability, behavioral disinhibition, alcohol consumption, AUD symptoms, and associated conduct problems via PTSD symptoms among those with the low expression 5-HTTLPR alleles. However, interactions between combat trauma and either childhood trauma or genotype were not significant. The results support
\end{abstract}


the hypothesis that affect lability and behavioral disinhibition are potential intermediate traits with distinct associations with AUD and associated externalizing problems.

Keywords: alcohol use disorder, affect dysregulation, PTSD, SLC6A4, rs25531

Posttraumatic stress disorder (PTSD) is a debilitating condition that affects OEF (Operation Enduring Freedom)/OIF (Operation Iraqi Freedom) veterans (Hoge, Auchterlonie, \& Milliken, 2006). Prevalence of PTSD among OEF/OIF veterans has increased substantially since 2002 to a current estimate of 23\% (Fulton et al., 2015; Seal et al., 2009). PTSD diagnosis increases the odds of alcohol use disorder (AUD) 4 -fold (Seal et al., 2011); 41\%-79\% of those with PTSD have an AUD (Hoge et al., 2004; Pietrzak, Goldstein, Southwick, \& Grant, 2011; Scherrer et al., 2008). Patients with comorbid PTSD and substance use disorder (SUD) are at a greater risk for health problems, suicide attempts, violent behavior, and legal problems (Driessen et al., 2008; Tate, Norman, McQuaid, \& Brown, 2007). Patients with PTSD and comorbid SUD are less likely to benefit from substance use treatment and more likely to relapse compared to those with SUD alone (Ouimette, Brown, \& Najavits, 1998). Given the social, health, and economic impact associated with PTSD and AUD, understanding risk mechanisms is critical for advancing prevention and treatment efforts. Environmental, genetic, temperament, and social-cognitive factors each act as predisposing, precipitating, or maintaining factors in the development and expression of symptoms and behavior. Research on the etiology and treatment of comorbid PTSD and AUD can be advanced by an integrative approach that includes multiple important domains. This study integrates the study of environmental factors (i.e., traumatic stress), genetic factors that contribute to stress reactivity, and individual differences in behavioral and emotional regulation.

\section{Mediators of associations between PTSD symptoms and externalizing problems}

AUD and other externalizing behaviors are hypothesized to be associated with different regulatory deficits, with behavioral disinhibition underlying conduct problems and affect lability conferring risk for dependence syndrome (Simons, Carey, \& Wills, 2009; Simons, Oliver, et al., 2005b; Simons, Wills, \& Neal, 2014). The affective processing model of negative reinforcement posits that fluctuation in negative affect is the core mechanism driving substance dependence (Baker, Piper, McCarthy, Majeskie, \& Fiore, 2004; McCarthy, Curtin, Piper, \& Baker, 2010). Given the importance of sensitization to negative affective states, high levels of negative affect, and rapid changes in negative affect in this model, we propose that individuals characterized by high levels of affective lability are at increased risk for the compulsive drinking patterns characteristic of AUD. PTSD is associated with pronounced emotional dysregulation. We posit that labile affect will mediate associations between PTSD and dependence syndrome symptoms.

Disinhibition has been linked to alcohol use and associated conduct problems (e.g., assault, interpersonal conflict; Carver, 2005; Fernie et al., 2013; Giancola, Parrott, \& Roth, 2006; Sher \& Trull, 1994; Wills et al., 2013). Disinhibition mediates associations between PTSD and alcohol-related problems (Miller, Vogt, Mozley, Kaloupek, \& Keane, 2006), predicts externalizing problems over and above the effects of alcohol consumption (Simons et 
al., 2009, 2005a), and moderates the relationship between alcohol use and conduct problems, increasing the strength of associations (Neal \& Carey, 2007; Simons, Gaher, et al., 2005). Hence, we hypothesize that disinhibition will mediate associations between PTSD symptoms and conduct related problems.

\section{Gene-by-environment $(G \times E)$ interactions}

Individual differences in the risk for developing psychiatric disorders, including PTSD and AUD, are likely the product of an individual's genetic predisposition and exposure to environmental risk (e.g., stress; Ducci \& Goldman, 2008; Koenen, Nugent, \& Amstadter, 2008; Sher et al., 2010; Tarter, 2002). There has been substantial interest in characterizing such genetic vulnerabilities, yet the findings have been mixed. A critical barrier to progress in this area has been the focus on categorical diagnoses as phenotypes. We hypothesize that individuals who carry the low expression "S" allele of the serotonin transporter (SLC6A4) regulatory region polymorphism (5-HTTLPR) will exhibit stronger associations between traumatic stress and PTSD symptoms. This is consistent with previous findings on 5-HTTLPR and PTSD (Kimbrel et al., 2015; Liu et al., 2015; Pietrzak, Galea, Southwick, \& Gelernter, 2013; Wald et al., 2013; Walsh, Uddin, Soliven, Wildman, \& Bradley, 2014). Results of two recent meta-analyses found limited evidence of direct effects of 5-HTTLPR on PTSD, indicate that effects are limited to individuals with high degrees of trauma exposure, and call for more research on $\mathrm{G} \times \mathrm{E}$ interactions (Gressier et al., 2013; Navarro-Mateu, Escamez, Koenen, Alonso, \& Sanchez-Meca, 2013). The present study seeks to advance previous work by testing moderating effects of the serotonin transporter (SLC6A4) regulatory region polymorphism on associations between both childhood traumatic stress and combat related traumatic stress and a continuous latent PTSD factor in a sample of veterans. Further, to better genetically characterize the expression (i.e. transcriptional efficiency) of 5-HTTLPR we also genotyped rs25531 and utilized the "triallelic" coding scheme, whereby "low" expression is indicated by carrying one or more copies of 5-HTTLPR "S" or rs25531 LG (Nakamura, Ueno, Sano, \& Tanabe, 2000).

\section{Stress sensitization versus stress inoculation}

Theoretical models on the role of premorbid stress in traumatic stress reactions have posited different relationships. These models vary in respect to the level of premorbid stress that is studied (i.e., manageable stressors versus severe stressors) and the nature of the effect (i.e., buffering versus vulnerability). The stress inoculation model (i.e., early exposure to manageable stressors) suggests that early life stress, especially experiences that are not overwhelming, may act as a protective factor making individuals more resilient to later stressful events (Fergus \& Zimmerman, 2005; Garmezy, 1991; Khoshaba \& Maddi, 1999; Masten, 2001; Mortimer \& Staff, 2004). Animal models have demonstrated early stress inoculation induced resiliency in monkeys (Lyons \& Parker, 2007) and rats (Meaney \& Szyf, 2005) by reducing their behavioral and hormonal responses to stress in adult life. In contrast, traumatic life stress taxes the development of an adaptive stress response system, making individuals more vulnerable to the effects of later life stress (Dougherty, Klein, \& 
Davila, 2004; Harkness, Bruce, \& Lumley, 2006; Kendler, Kuhn, \& Prescott, 2004; Shapero et al., 2014). Exposure to extreme stress early in life leads to undesirable outcomes (e.g., anxiety, depression) through the interaction between genetic vulnerability and neural circuits responsible for emotion regulation (Gillespie, Phifer, Bradley, \& Ressler, 2009). In the current study, we focus on the vulnerability model and test whether childhood traumatic stress potentiates the association between combat stress and PTSD among veterans to better characterize the associations between combat stress and PTSD symptoms.

\section{Summary}

The proposed model is depicted in Figure 1. Gender, combat stress, childhood stress, serotonin transporter 5-HTTLPR/rs25531 genotype, and the stress and genotype interactions were exogenous variables predicting a latent PTSD severity indicator. PTSD symptoms, in turn, predicted alcohol use, affect lability, and disinhibition. Associations between PTSD symptoms and symptoms of alcohol dependence syndrome were expected to be indirect via alcohol consumption and lability. Associations between PTSD symptoms and conduct problems were expected to be indirect via alcohol consumption and disinhibition. Gender was included as a covariate due its potential associations with the outcomes. Men tend to be at higher risk for externalizing problems (e.g., AUD; Hicks et al., 2007). In contrast, research on gender differences in affect lability (Samuel, South, \& Griffin, 2015; Simons et al., 2014) and PTSD (Jacobson, Donoho, Crum-Cianflone, \& Maguen, 2015) has been more equivocal.

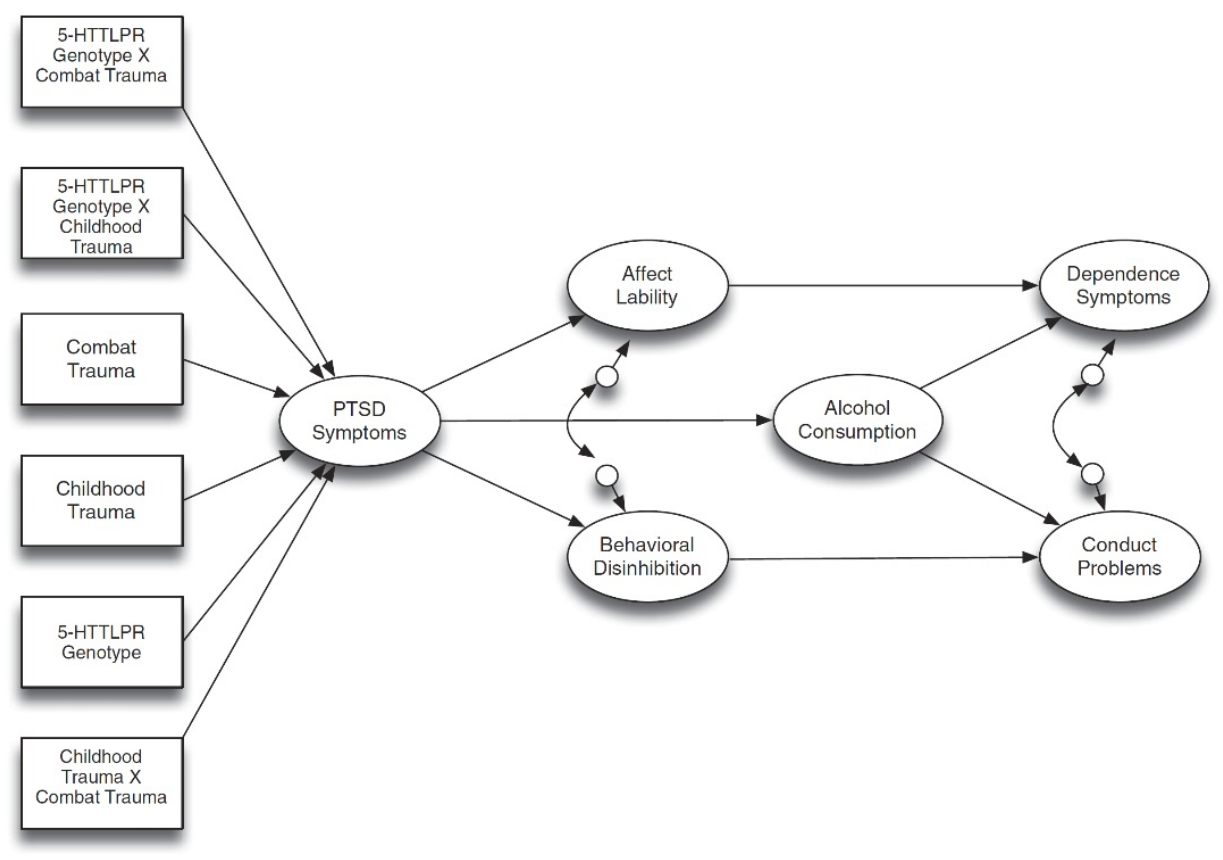

Figure 1. Hypothesized model. Gender is included with paths to all endogenous variables but is omitted for clarity. 


\section{Method}

\subsection{Participants}

Participants were 270 OIF/OEF/OND (Operation New Dawn) veterans age 21-51 ( $M=33.25$, $S D=6.59$ ). Thirteen percent were women. The sample was $81 \%$ white, $10 \%$ black, $3 \%$ multiracial, $1 \%$ Asian, and $5 \%$ other races or did not respond. Seven percent were Hispanic. The median number of deployments was 2. Approximately $47 \%$ were in the National Guard or Reserves when called up for their first deployment. Most served in the Army (64\%), followed by Air Force (12\%), Marines (10\%), Navy (6\%), and Coast Guard (1\%). The remainder specified the National Guard but not a military branch.

\subsection{Measures}

\subsubsection{PTSD symptoms}

The 17-item National Center for PTSD Checklist - Military Version was used to assess PTSD symptoms (Blanchard, Jones-Alexander, Buckley, \& Forneris, 1996; Weathers, Litz, Herman, Huska, \& Keane, 1993). The PCL-M items correspond to DSM-IV criteria and symptoms and are rated on a 5-point scale. The scale is internally consistent $(\alpha=0.97$ in the current sample) and has good sensitivity (0.82) and specificity (0.83) in predicting PTSD diagnoses among veterans (Weathers et al., 1993).

\subsubsection{Combat and childhood traumatic stress}

The Deployment Risk and Resilience Inventory - 2 (DRRI-2; Vogt, Smith, King, \& King, 2012)was used to assess traumatic stress. The combat experiences subscale consists of 17 items rated on 6-point scales $(1=$ Never, 6 = Daily or almost daily). Sample items include, "I was exposed to hostile incoming fire" and "I personally witnessed someone from my unit or an ally unit being seriously wounded or killed." Cronbach's alpha in the current sample was 0.95 . Childhood trauma was assessed by 5 dichotomous items from the predeployment stressors subscale. The items assessed interpersonal traumatic stress that occurred before age 18. Items include: (1) "I saw or heard physical fighting between my parents or caregivers," (2) "I was physically punished by a parent or primary caregiver," (3) "I experienced unwanted sexual activity as a result of force, threat of harm, or manipulation during childhood (before age 18)," (4) "I was emotionally mistreated (for example, ignored or repeatedly told I was no good)," and (5) "I was seriously physically injured by another person (for example, hit or beaten up) during childhood (before age 18)." Cronbach's alpha in the current sample was 0.72 .

\subsubsection{Affect lability}

Lability was a latent variable comprised of three indicators representing the subscales of the Affective Lability Scales - Short form (Oliver \& Simons, 2004). The subscales reflect affective lability in respect to vacillating between depression and elation (8 items, $\alpha=0.91$ ), anxiety and depression ( 5 items, $\alpha=0.91$ ), and anger and euthymic mood ( 5 items, $\alpha=0.89$ ). 


\subsubsection{Disinhibition}

Disinhibition was a latent variable comprised of three indicators reflecting impulsivity (5 items, $\alpha=0.82$; (Eysenck, Pearson, Easting, \& Allsopp, 1985), distractibility (6 items, $\alpha=0.85$; (Kendall \& Williams, 1982), and impatience (3 items, $\alpha=0.70$; (Kendall \& Williams, 1982). These subscales load on a replicable latent factor of poor behavioral control in previous research (Simons et al., 2009; Simons, Wills, Emery, \& Spelman, 2015; Wills, Pokhrel, Morehouse, \& Fenster, 2011).

\subsubsection{Alcohol use}

Quantity of alcohol use in the past 90 days was assessed by the Modified Daily Drinking Questionnaire consisting of a grid representing the 7 days of the week (Dimeff, Baer, Kivlahan, \& Marlatt, 1999). Participants were provided definitions of standard drinks and reported the number of drinks they consumed for each day in a typical week. Weekly consumption was calculated by summing the number of standard drinks across the days. Frequency of alcohol use in the past 90 days was assessed by a 9-point anchored rating scale $(0=$ no use, $8=$ more than once a day). These two indicators were used as indicators of a latent alcohol consumption factor.

\subsubsection{Alcohol dependence symptoms}

The Alcohol Dependence Scale (ADS; Skinner \& Allen, 1982; Skinner \& Horn, 1984) was used to assess symptoms of alcohol dependence syndrome. The scale contains 25 items assessing loss of control over drinking ( $\alpha=82)$, obsessive-compulsive drinking style $(\alpha=63)$, and psychophysical withdrawal $(\alpha=0.75)$ and exhibits good convergent validity with other indicators of AUD (Doyle \& Donovan, 2009). Scale anchors vary across item. Three subscales were used as indicators of a latent alcohol dependence syndrome factor.

\subsubsection{Conduct problems}

Three subscales of the Drinkers Inventory of Negative Consequences (Miller, Tonigan, \& Longabaugh, 1995) were used as indicators of a latent conduct problems factor. The social responsibility subscale contains 7 items assessing failure to fulfill role responsibilities ( $\alpha=$ 0.88). The interpersonal factor contains 10 items assessing problems with interpersonal functioning $(\alpha=0.91)$. The impulse control/risk behavior factor contains 12 items assessing behavioral dyscontrol associated with drinking $(\alpha=0.84)$.

\subsection{Procedure}

OIF/OEF/OND veterans were recruited from two communities (Tampa Bay area, Florida, and Vermillion/Sioux Falls, South Dakota). Participants were recruited from the local Veteran Affairs Medical Centers, local universities, and surrounding communities via newspaper advertisements, flyers, mail correspondence, and clinician referral. Participants completed self-report surveys and provided a saliva sample for genotyping. All procedures were approved by the respective institutional review boards. Participants were paid $\$ 25$ to participate. Participation was voluntary and responses were confidential.

Saliva samples were collected using Oragene-DNA (OG-500) DNA collection kits (DNA Genotek, Ontario, Canada). DNA was extracted from saliva using the PURGENE DNA 
Isolation Kit (Gentra Systems, Inc., Minneapolis, Minnesota, USA) according to the manufacturer's instructions. Genotypes for 5-HTTLPR and rs25531 were assessed by polymerase chain reaction (PCR) with forward and reverse primers: $5^{\prime}$-TCCTCCGCTTTGGCGCCTCT TCC-3' and 5'-TGGGGGTTGCAGGGGAGATCCTG-3' (Wendland, Martin, Kruse, Lesch, $\&$ Murphy, 2006). The PCRs were performed in 25- $\mu$ l reactions containing $20 \mathrm{ng}$ of DNA, $1 \times$ GoTaq Master Mix (Promega, Madison, Wisconsin, USA), and $10 \mu \mathrm{M}$ of each primer. The rs25531 polymorphism was recognized by digestion with HpaII with 1× BSA (New England BioLabs, Ipswich, Massachusetts, USA) overnight at $37^{\circ} \mathrm{C}$ using $15 \mu \mathrm{l}$ of the PCR product. Products were separated by electrophoresis on a $2.5 \%$ agarose gel and visualized under UV light with SybrSafe stain. A randomly selected subset of the sample (10\%) was rerun for each polymorphism to check for genotype call discrepancies. No discrepancies were found.

\section{Results}

\subsection{Descriptive statistics}

Descriptive statistics are presented in Table 1 and bivariate correlations are presented in Table 2. Two-thirds of the sample exceeded a cut score of 36 on the PCL, suggesting a potential PTSD diagnosis and $43 \%$ exceeded a more conservative cut score of 50 recommended for Veteran Affairs (VA) specialty clinics (U.S. Department of Veteran Affairs, 2012). On the ADS, approximately $37 \%$ scored 9 or higher, suggesting a potential AUD (Skinner \& Horn, 1984). These statistics, in conjunction with those presented in Table 1, indicate that the sample consisted of a wide range of alcohol involvement and PTSD symptoms with a considerable proportion endorsing clinically significant symptoms. Consistent with previous research in predominantly white samples, approximately $66 \%$ of the sample carried the low expression alleles of 5-HTTLPR (S or LG). Allele frequencies observed for 5-HTTLPR were $\mathrm{L}=0.62, \mathrm{~S}=0.38$ and $\mathrm{XL}=0.004$. The observed genotype frequencies $\mathrm{L} / \mathrm{L}$ $=118, \mathrm{~L} / \mathrm{S}=96, \mathrm{~S} / \mathrm{S}=54, \mathrm{XL} / \mathrm{L}=1, \mathrm{XL} / \mathrm{S}=1$ were not in Hardy-Weinberg equilibrium $\left(\chi^{2}=\right.$ $15.47, p<0.001$ ). A randomly selected $10 \%$ of the sample was re-genotyped, and no call discrepancies were identified. We are unable to determine the cause of the departure from expectations $(\mathrm{L} / \mathrm{L}=102.82, \mathrm{~L} / \mathrm{S}=126.36, \mathrm{~S} / \mathrm{S}=38.82)$. Allele frequencies observed for rs25531 were $A=0.922$ and $G=0.078$. The observed genotype frequencies 3 were in Hardy-Weinberg equilibrium $\left(\chi^{2}=1.35, p=0.246\right)$. Genotype was not related to either gender $\left(\chi^{2}(1, N=270)\right.$ $=1.52, p=0.217$ ) or race (white vs. nonwhite; $\left.\chi^{2}(1, N=268)=0.62, p=0.609\right)$. Combat trauma exhibited moderate to strong correlations with PTSD symptoms and affect lability, and slightly weaker associations with disinhibition and conduct problems. Associations between childhood trauma and the outcomes followed a similar pattern but were weaker. PTSD symptoms exhibited moderate to strong correlations with indicators of lability, disinhibition, symptoms of AUD, and conduct problems. 
SimOnS ET AL., ADDICTIVE BEHAVIORS 64 (2017)

Table 1. Descriptive statistics

\begin{tabular}{lrrrrr}
\hline Variable & \multicolumn{1}{c}{ M } & \multicolumn{1}{c}{$S D$} & Min & Max & Skew \\
\hline Combat trauma & 34.41 & 17.79 & 17.00 & 92.00 & 1.36 \\
Childhood trauma & 0.97 & 1.35 & 0.00 & 5.00 & 1.38 \\
PCL-hyperarousal & 3.18 & 1.20 & 1.00 & 5.00 & -0.19 \\
PCL-intrusion & 2.59 & 1.17 & 1.00 & 5.00 & 0.42 \\
PCL-avoidance & 2.64 & 1.19 & 1.00 & 5.00 & 0.26 \\
Impulsivity & 2.19 & 0.88 & 1.00 & 5.00 & 0.89 \\
Impatience & 1.79 & 0.75 & 1.00 & 5.00 & 1.33 \\
Distractibility & 2.42 & 0.87 & 1.00 & 5.00 & 0.51 \\
Lability (anx./dep.) & 2.12 & 0.89 & 1.00 & 4.00 & 0.25 \\
Lability (dep./ela.) & 2.16 & 0.78 & 1.00 & 4.00 & 0.26 \\
Lability (anger) & 2.05 & 0.84 & 1.00 & 4.00 & 0.49 \\
Weekly drinks & 15.58 & 15.17 & 0.00 & 76.00 & 1.88 \\
Alcohol frequency & 4.45 & 1.57 & 0.00 & 8.00 & -0.22 \\
ADS_LC & 3.92 & 3.33 & 0.00 & 15.00 & 0.90 \\
ADS_OC & 1.27 & 1.64 & 0.00 & 8.00 & 1.41 \\
ADS_WD & 2.34 & 2.78 & 0.00 & 16.00 & 1.62 \\
Interpersonal functioning & 3.46 & 4.82 & 0.00 & 29.00 & 2.41 \\
Social responsibility & 2.00 & 3.12 & 0.00 & 20.00 & 2.45 \\
Risk behavior & 4.99 & 5.33 & 0.00 & 30.00 & 1.83 \\
\hline Note $N$ s range from & & & & & \\
\hline
\end{tabular}

Note: $N^{\prime}$ s range from 258 to 270 due to missing data. PCL = PTSD checklist. Anx. = Anxiety, Dep. = Depression, Ela. $=$ Elation, ADS_LC $=$ Alcohol Dependence Scale-Loss of control, ADS_OC $=$ Alcohol Dependence ScaleObsessive compulsive, ADS_WD = Alcohol Dependence Scale-Withdrawal 


\begin{tabular}{|c|c|c|c|c|c|c|c|c|c|c|c|c|c|c|c|c|c|c|c|c|}
\hline & 1 & 2 & 3 & 4 & 5 & 6 & 7 & 8 & 9 & 10 & 11 & 12 & 13 & 14 & 15 & 16 & 17 & 18 & 19 & 20 \\
\hline 1. Gender & - & & & & & & & & & & & & & & & & & & & \\
\hline 2. Genotype & -0.08 & - & & & & & & & & & & & & & & & & & & \\
\hline 3. Combat trauma & $0.13^{*}$ & -0.11 & - & & & & & & & & & & & & & & & & & \\
\hline 4. Child trauma & $-0.14^{*}$ & -0.00 & $0.12^{*}$ & - & & & & & & & & & & & & & & & & \\
\hline 5. $\mathrm{PCL} \_$arousal & 0.03 & -0.04 & $0.45^{* * *}$ & $0.25^{* * *}$ & - & & & & & & & & & & & & & & & \\
\hline 6. $\mathrm{PCL}$ intrusion & -0.03 & -0.05 & $0.56^{* * *}$ & $0.22^{* * *}$ & $0.78^{* * *}$ & - & & & & & & & & & & & & & & \\
\hline 7. $\mathrm{PCL} \_$avoidance & -0.04 & -0.06 & $0.47^{* * *}$ & $0.24^{* * *}$ & $0.85^{* * *}$ & $0.84^{* * *}$ & - & & & & & & & & & & & & & \\
\hline 8. Impulsivity & 0.03 & $-0.15^{*}$ & $0.25^{* * *}$ & $0.13^{*}$ & $0.30^{* * *}$ & $0.31^{* * *}$ & $0.35^{* * *}$ & - & & & & & & & & & & & & \\
\hline 9. Impatience & -0.02 & -0.08 & $0.17^{* *}$ & 0.11 & $0.32^{* * *}$ & $0.29 * * *$ & $0.35^{* * *}$ & $0.55^{* * *}$ & - & & & & & & & & & & & \\
\hline 10. Distractibility & -0.08 & -0.10 & $0.18^{* *}$ & $0.17^{* *}$ & $0.38^{* * *}$ & $0.34^{* * *}$ & $0.39^{* * *}$ & $0.48^{* * *}$ & $0.49^{* * *}$ & - & & & & & & & & & & \\
\hline 11. ALS-Anx/Dep & -0.04 & $-0.13^{*}$ & $0.35^{* * *}$ & $0.20^{* *}$ & $0.64^{* * *}$ & $0.61^{* * *}$ & $0.66^{* * *}$ & $0.36^{* * *}$ & $0.39^{* * *}$ & $0.48^{* * *}$ & - & & & & & & & & & \\
\hline 12. ALS-Dep/Ela & 0.01 & -0.10 & $0.29^{* * * *}$ & $0.25^{* * *}$ & $0.56^{* * *}$ & $0.51^{* * *}$ & $0.59^{* * *}$ & $0.38^{* * *}$ & $0.42^{* * *}$ & $0.53^{* * * *}$ & $0.76^{* * * *}$ & - & & & & & & & & \\
\hline 13. ALS-anger & 0.04 & $-0.14^{*}$ & $0.36^{* * *}$ & $0.23^{* * *}$ & $0.61^{* * *}$ & $0.57^{* * *}$ & $0.61^{* * *}$ & $0.45^{* * *}$ & $0.37^{* * *}$ & $0.40^{* * *}$ & $0.69^{* * *}$ & $0.68^{* * *}$ & - & & & & & & & \\
\hline 14. Weekly drinks & 0.07 & 0.09 & $0.14^{*}$ & 0.08 & $0.33^{* * *}$ & $0.24^{* * *}$ & $0.30^{* * *}$ & $0.13^{*}$ & 0.10 & 0.10 & $0.19^{* *}$ & $0.20^{* *}$ & $0.17^{* * *}$ & - & & & & & & \\
\hline 15. Alcohol freq. & 0.07 & 0.09 & 0.03 & 0.08 & $0.17^{* *}$ & $0.13^{*}$ & $0.13^{*}$ & 0.02 & 0.06 & -0.00 & 0.10 & 0.09 & 0.05 & $0.61^{* * *}$ & - & & & & & \\
\hline 16. ADS-LC & 0.03 & -0.04 & $0.16^{* *}$ & 0.10 & $0.28^{* * *}$ & $0.30^{* * *}$ & $0.35^{* * *}$ & $0.22^{* * * *}$ & $0.23^{* * *}$ & $0.24^{* * *}$ & $0.29^{* * *}$ & $0.27^{* * *}$ & $0.22^{* * *}$ & $0.49^{* * *}$ & $0.26^{* * *}$ & - & & & & \\
\hline 17. ADS-OC & 0.10 & 0.02 & $0.18^{* *}$ & 0.03 & $0.34^{* * *}$ & $0.33^{* * *}$ & $0.41^{* * * *}$ & $0.27^{* * *}$ & $0.22^{* * *}$ & $0.20^{* *}$ & $0.37^{* * *}$ & $0.38^{* * *}$ & $0.34^{* * *}$ & $0.52^{* * *}$ & $0.38^{* * *}$ & $0.62^{* * *}$ & - & & & \\
\hline 18. ADS-WD & 0.04 & -0.01 & $0.16^{*}$ & $0.20^{* * *}$ & $0.41^{* * *}$ & $0.39^{* * * *}$ & $0.45^{* * *}$ & $0.26^{* * *}$ & $0.31^{* * *}$ & $0.28^{* * * *}$ & $0.47^{* * *}$ & $0.41^{* * * *}$ & $0.42^{2 * * *}$ & $0.35^{* * * *}$ & $0.28^{* * *}$ & $0.58^{* * *}$ & $0.59^{* * * *}$ & - & & \\
\hline 19. Interpersonal & 0.07 & -0.07 & $0.23^{* * *}$ & $0.15^{*}$ & $0.34^{* * *}$ & $0.34^{* * *}$ & $0.40^{* * * *}$ & $0.32^{2 * * *}$ & $0.32^{* * *}$ & $0.24^{* * *}$ & $0.37^{* * *}$ & $0.38^{* * *}$ & $0.36^{* * *}$ & $0.37^{* * *}$ & $0.21^{* * *}$ & $0.59^{* * * *}$ & $0.60^{* * *}$ & $0.57^{* * *}$ & - & \\
\hline 20. Social resp. & 0.08 & -0.06 & $0.25^{* * *}$ & 0.10 & $0.34^{* * *}$ & $0.35^{* * *}$ & $0.41^{* * *}$ & $0.27^{* * *}$ & $0.26^{* * *}$ & $0.25^{* * *}$ & $0.36^{* * * *}$ & $0.38^{* * *}$ & $0.33^{* * * *}$ & $0.43^{* * *}$ & $0.21^{* * *}$ & $0.61^{* * *}$ & $0.63^{* * *}$ & $0.55 * *$ & $0.85^{* * * *}$ & - \\
\hline 21. Risk behavior & $0.13^{*}$ & -0.08 & $0.25^{* * *}$ & $0.13^{*}$ & $0.37^{* * *}$ & $0.40^{* * *}$ & $0.43^{* * *}$ & $0.30^{* * *}$ & $0.28^{* * *}$ & $0.22^{* * *}$ & $0.35^{* * *}$ & $0.35^{* * *}$ & $0.36^{* * * *}$ & $0.45^{* * *}$ & $0.25^{* * *}$ & $0.63^{* * *}$ & $0.63^{* * *}$ & $0.57 * * *$ & $0.80^{* * * *}$ & $0.83^{* * *}$ \\
\hline
\end{tabular}

Note: $N^{\prime}$ s range from 248 to 270 due to missing data. Gender is coded $0=$ women, $1=$ men. Genotype is coded $0=$ high expression alleles of 5-HTTLPR $\left(\mathrm{L}_{A}\right), 1=$ low expression alleles of 5-HTTLPR $(\mathrm{S}$ or Lc). PCL $=$ PTSD checklist, ALS $=$ Affect lability Scale, ADS = Alcohol Dependence Scale, Freq = Frequency, Anx. = Anxiety, Dep. $=$ Depression. Ela. $=$ Elation. ADS_LC = Alcohol Dependence Scale - Loss of control, ADS_OC = Alcohol Dependence Scale - Obsessive compulsive, Alcohol Dependence Scale - Withdrawa

$* p<0.05$

$* * p<0.01$
${ }^{* * * *} p<0.001$ 


\subsection{Measurement model}

The hypothesized measurement model for the latent constructs was tested in Mplus 7.4 with the maximum likelihood robust (MLR) estimator (Muthén \& Muthén, 2015). The model fit well $\chi^{2}(104, N=270)=147.37, p=0.003, \mathrm{CFI}=0.98$, RMSEA $=0.03990 \% \mathrm{CI}[0.023$, $0.053], \mathrm{SRMR}=0.036$. Standardized factor loadings ranged from $0.62-0.99, p^{\prime} \mathrm{s}<0.001$.

\subsection{Structural model}

The combat trauma and childhood trauma scales were mean centered prior to forming the interactions (Aiken \& West, 1991). The hypothesized structural model depicted in Figure 1 was tested in Mplus 7.4 with the MLR estimator (Muthén \& Muthén, 2015). Neither the combat trauma $\times$ genotype nor combat trauma $\times$ childhood trauma interaction $\left(p^{\prime} s>0.30\right)$ were significant. These two nonsignificant interactions were dropped sequentially and the model reestimated to form a more parsimonious model. The final model was a good fit to the data $\chi^{2}(185, N=270)=253.34, p=0.001, \mathrm{CFI}=0.98$, RMSEA $=0.03790 \% \mathrm{CI}[0.025,0.048]$, $\mathrm{SRMR}=0.052$. Following fitting the model, we examined Cook's D, influence, and the loglikelihood contribution of the observations. Five observations that could have potential undue influence on the model were examined. However, estimating the model without these observations had no appreciable effect on the significance of the path coefficients or interaction, and hence the full sample was retained. ${ }^{1}$ The model is depicted in Figure 2. Gender was included with paths estimated to each endogenous construct. There was a marginal association between male gender and alcohol consumption $(\beta=0.11(S E=0.06)$, $p=0.052)$. None of the other gender effects approached significance $\left(p^{\prime} \mathrm{s}>0.28\right)$. Consistent with hypothesis, there was a significant interaction between childhood trauma and genotype $(\beta=0.24(S E=0.11), p=0.034)$. The simple slopes indicated that childhood trauma was positively associated with PTSD symptoms for individuals carrying the low expression alleles of 5-HTTLPR ( $S$ or LG; $\beta=0.27(S E=0.07), p<0.001$ ) but not for those with the high expression alleles of 5-HTTLPR (LA; $\beta=0.03(S E=0.09), p=0.724)$. Hence, the effect of childhood trauma on PTSD symptoms increases 8-fold for those with the low-expression allele (see Fig. 3). 


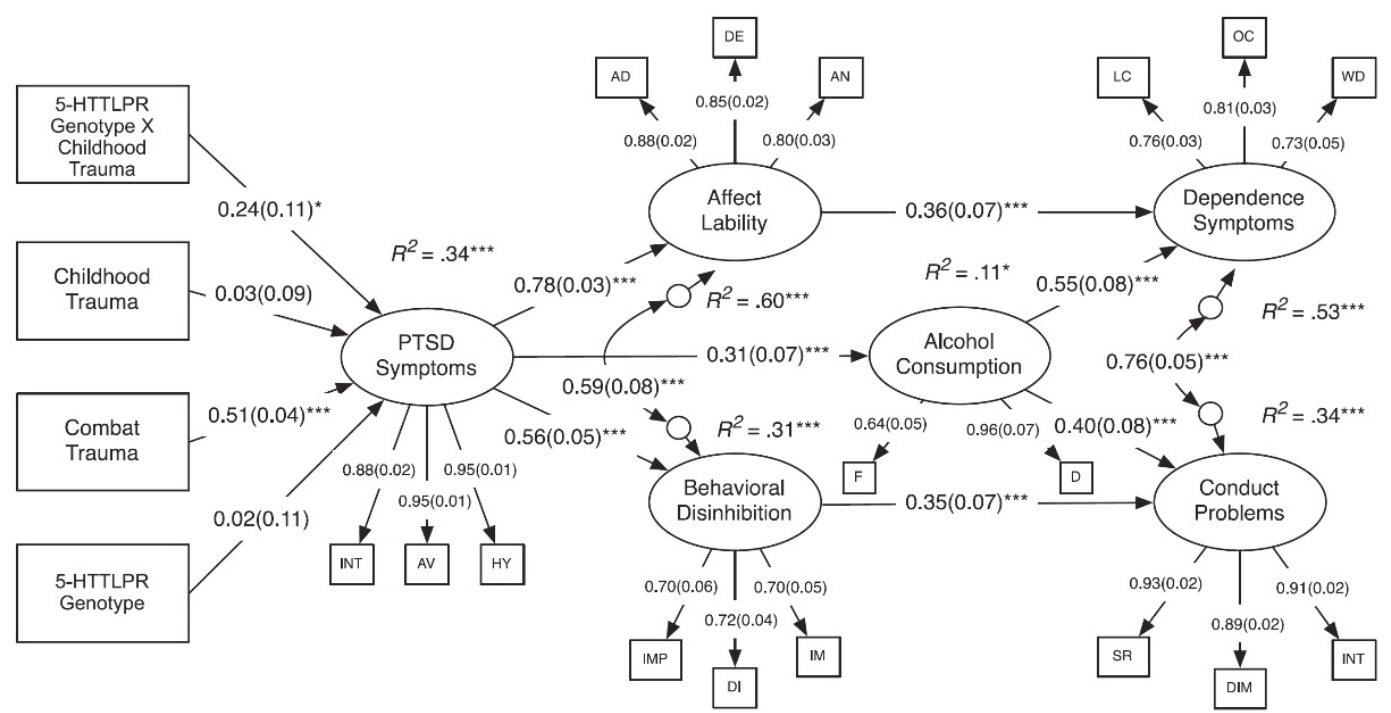

Figure 2. Final model. $\chi^{2}(185, N=270)=253.34, p=0.001, \mathrm{CFI}=0.98, \mathrm{RMSEA}=0.03790 \%$ CI $[0.025,0.048]$, SRMR $=0.052$. Coefficients are standardized. Standard errors are in parentheses. Gender is included with paths to all endogenous variables but is omitted for clarity. PTSD indicators: INT = intrusion, AV = avoidance, HY = hyperarousal. Lability indicators: $\mathrm{AD}=$ anxiety-depression, $\mathrm{DE}=$ depression-elation, $\mathrm{AN}=$ anger. Disinhibition indicators: $\mathrm{IMP}=$ impulsivity, $\mathrm{DI}=$ distraction, $\mathrm{IM}=$ impatience. Alcohol indicators: $\mathrm{F}=$ frequency, $\mathrm{D}=$ drinks per week. Dependence indicators: $\mathrm{LC}=\mathrm{Loss}$ of control, $\mathrm{OC}=\mathrm{Ob}-$ sessive-compulsive, $\mathrm{WD}=$ withdrawal. Conduct indicators (DrInC subscales): $\mathrm{SR}=$ social responsibility, DIM $=$ Impulse/risk behavior, $\mathrm{INT}=$ interpersonal. ${ }^{*} p<0.05,{ }^{* * *} p<0.001$.

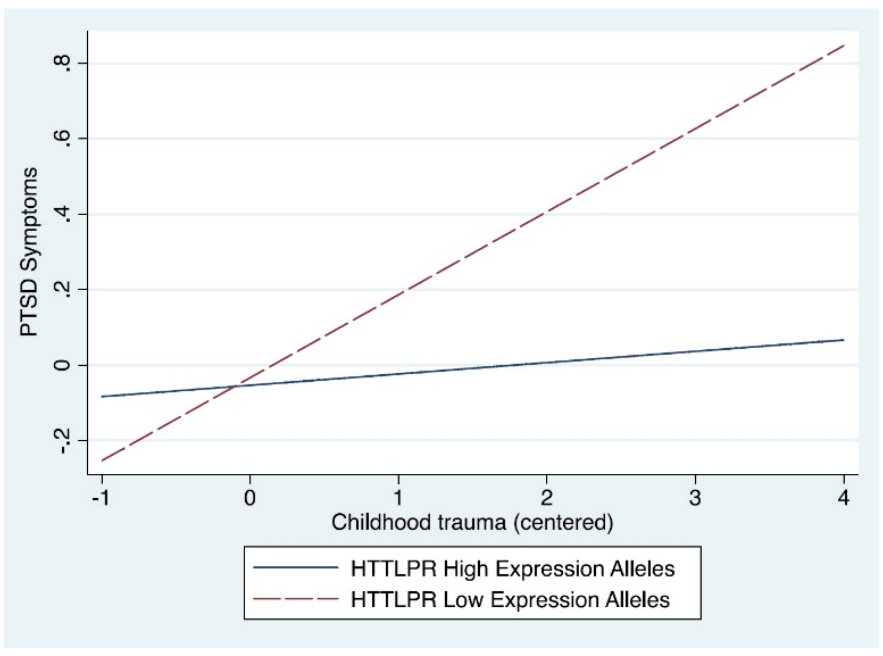

Figure 3. $G \times E$ interaction showing conditional effects of childhood trauma on PTSD symptoms as a function of genotype at the serotonin transporter linked polymorphic region (5-HTTLPR). 


\subsubsection{Indirect effects}

The indirect effects are presented in Tables 3-4. Consistent with hypothesis, PTSD symptoms mediated associations between combat trauma and both lability and disinhibition. For childhood trauma, the indirect effects were significant for those with the low expression alleles of 5-HTTLPR (S or LG) but not the high expression alleles of 5-HTTLPR (LA). Also consistent with hypothesis, affect lability mediated associations between PSTD symptoms and symptoms of DSM-IV alcohol dependence syndrome. Disinhibition mediated associations between PTSD symptoms and conduct problems. In addition, combat trauma was indirectly associated with alcohol consumption, dependence symptoms, and conduct problems via its effects on PTSD symptoms. Finally, there were significant conditional indirect effects of childhood trauma on the outcomes for those carrying the low expression alleles of 5-HTTLPR.

Table 3. Indirect effects in predicting lability and disinhibition

\begin{tabular}{lccccc}
\hline & \multicolumn{2}{c}{ Lability } & & \multicolumn{2}{c}{ Disinhibition } \\
\cline { 2 - 3 } \cline { 5 - 6 } Predictor & Indirect effect & $95 \% \mathrm{CI}$ & & Indirect effect & $95 \% \mathrm{CI}$ \\
\hline Combat trauma & $\mathbf{0 . 4 0}$ & {$[\mathbf{0 . 2 2}, \mathbf{0 . 4 7}]$} & & $\mathbf{0 . 2 8}$ & {$[\mathbf{0 . 2 1}, \mathbf{0 . 3 6}]$} \\
Childhood trauma (S, LG) & $\mathbf{0 . 2 1}$ & {$[\mathbf{0 . 1 1}, \mathbf{0 . 3 2}]$} & & $\mathbf{0 . 1 5}$ & {$[\mathbf{0 . 0 7}, \mathbf{0 . 2 3}]$} \\
Childhood trauma (LA) & 0.03 & {$[-0.12,0.17]$} & & 0.02 & {$[-0.09,0.12]$} \\
\hline
\end{tabular}

Note: $N=270$. Effects are standardized. Ind. = Indirect effect. S, LG, LA reflect conditional indirect effects for carriers of the low- and high-expression alleles of 5-HTTLPR. Bias-corrected bootstrapped confidence intervals were used to determine the significance of effects (MacKinnon, Lockwood, \& Williams, 2004). Bold font $=$ Significant effect based on $95 \%$ confidence interval.

\begin{tabular}{|c|c|c|c|c|c|c|c|}
\hline \multirow[b]{2}{*}{ Predictor } & \multirow{2}{*}{$\begin{array}{l}\text { Specific } \\
\text { Mediator }\end{array}$} & \multicolumn{2}{|c|}{ Alcohol consumption } & \multicolumn{2}{|c|}{ Dependence symptoms } & \multicolumn{2}{|c|}{ Conduct problems } \\
\hline & & Ind. & Tot. & Ind. & Tot. & Ind. & Tot. \\
\hline Combat trauma & - & $\begin{array}{c}0.16 \\
{[0.09,0.23]}\end{array}$ & $\begin{array}{c}0.16 \\
{[0.09,0.23]}\end{array}$ & $\begin{array}{c}0.23 \\
{[0.16,0.30]}\end{array}$ & $\begin{array}{c}0.23 \\
{[0.16,0.30]}\end{array}$ & $\begin{array}{c}0.16 \\
{[0.11,0.22]}\end{array}$ & $\begin{array}{c}0.16 \\
{[0.11,0.22]}\end{array}$ \\
\hline Childhood trauma $(\mathrm{S}, \mathrm{LG})$ & - & $\begin{array}{c}0.09 \\
{[0.03,0.14]}\end{array}$ & $\begin{array}{c}0.09 \\
{[0.03,0.14]}\end{array}$ & $\begin{array}{c}0.12 \\
{[0.06,0.19]}\end{array}$ & $\begin{array}{c}0.12 \\
{[0.06,0.19]}\end{array}$ & $\begin{array}{c}0.09 \\
{[0.04,0.14]}\end{array}$ & $\begin{array}{c}0.09 \\
{[0.04,0.14]}\end{array}$ \\
\hline Childhood trauma (LA) & - & $\begin{array}{c}0.01 \\
{[-0.05,0.07]}\end{array}$ & $\begin{array}{c}0.01 \\
{[-0.05,0.07]}\end{array}$ & $\begin{array}{c}0.02 \\
{[-0.07,0.10]}\end{array}$ & $\begin{array}{c}0.02 \\
{[-0.07,0.10]}\end{array}$ & $\begin{array}{c}0.01 \\
{[-0.05,0.07]}\end{array}$ & $\begin{array}{c}0.01 \\
{[-0.05,0.07]}\end{array}$ \\
\hline PTSD & Alcohol cons. & & & $\begin{array}{c}0.17 \\
{[0.08,0.27]}\end{array}$ & $\begin{array}{c}0.45 \\
{[0.35,0.56]}\end{array}$ & $\begin{array}{c}0.13 \\
{[0.05,0.21]}\end{array}$ & $\begin{array}{c}0.32 \\
{[0.23,0.42]}\end{array}$ \\
\hline PTSD & Lability & & & $\begin{array}{c}0.28 \\
{[0.17,0.39]}\end{array}$ & $\begin{array}{c}0.45 \\
{[0.35,0.56]}\end{array}$ & & \\
\hline PTSD & Disinhibition & & & & & $\begin{array}{c}0.20 \\
{[0.10,0.29]}\end{array}$ & $\begin{array}{c}0.32 \\
{[0.23,0.22]}\end{array}$ \\
\hline
\end{tabular}

Note: $N=270$. Effects are standardized. $95 \%$ confidence intervals are below each effect. Ind. $=$ Indirect effect. Tot. $=$ Total effect. Cons. $=$ Consumption. S, LG, La reflect conditional indirect effects for carriers of the low and high expression alleles of 5-HTTLPR. Indirect effects for trauma are all of the indirect effects. Specific indirect pathways for PTSD are provided. Total effects combine all indirect pathways. Biascorrected bootstrapped confidence intervals were used to determine the significance of effects (MacKinnon, Lockwood, \& Williams, 2004). Bold font $=$ Significant effect based on $95 \%$ confidence interval. 


\section{Discussion}

This study of OIF/OEF/OND veterans tested a structural model linking childhood and combat traumatic stress to externalizing problems of alcohol dependence syndrome symptoms and conduct problems. Associations between traumatic stress and emotional and behavioral dysregulation (i.e., lability and disinhibition) were indirect via PTSD symptoms. Lability, in turn, was hypothesized to mediate associations between PTSD symptoms and dependence syndrome symptoms, whereas disinhibition was hypothesized to mediate associations between PTSD symptoms and conduct problems. Genotype at the serotonin transporter linked polymorphic region (5-HTTLPR/rs25531) was hypothesized to moderate associations between traumatic stress and PTSD symptoms. Finally, childhood trauma and combat trauma were hypothesized to interact to predict PTSD symptoms. The hypothesized model was largely supported. Findings are discussed below.

\section{1. $G \times E$ and $E \times E$ interactions}

Neither childhood trauma nor 5-HTTLPR genotype moderated associations between combat trauma and PTSD symptoms. However, the 5-HTTLPR genotype did moderate associations between childhood trauma and PTSD symptoms as expected. There was a significant association between childhood traumatic stress and PTSD only among those carrying the low expression alleles of the 5-HTTLPR. Hence, for those at risk, childhood trauma was associated with increased PTSD symptomatology after controlling for combat trauma. This association, along with the significant bivariate associations between childhood trauma and PTSD symptoms is consistent with previous research indicating that childhood adversity is associated with vulnerability to psychopathology in later life (Dougherty et al., 2004; Harkness et al., 2006; Kendler et al., 2004; Shapero et al., 2014). However, in the current study, the effect of childhood trauma on PTSD symptoms was additive. That is, childhood traumatic stress did not alter the association between combat trauma and PTSD symptoms as hypothesized. In addition, the effect of childhood traumatic stress on PTSD symptomology was limited to those who also exhibited genetic risk. Or, alternatively, the high expression alleles of 5-HTTPR served as a protective factor, reducing the effect of childhood traumatic stress on PTSD symptoms.

Previous research on associations between genotype at the serotonin transporter linked polymorphic region (5-HTTLPR) and PTSD symptoms has produced mixed results (Gressier et al., 2013; Navarro-Mateu et al., 2013; Wang et al., 2011). Research has indicated direct effects in non-Hispanic Black but not non-Hispanic White samples (Liu et al., 2015) whereas other studies have found stronger effects in veterans with European ancestry (Kimbrel et al., 2015). Research testing interactions between traumatic stress and variation in the 5-HTTLPR polymorphism has also been mixed (Liu et al., 2015; Pietrzak et al., 2013). Recent meta-analyses suggest that the effect of 5-HTTPLR genotype may be evident only in high trauma exposed samples (Gressier et al., 2013; Navarro-Mateu et al., 2013). Our results are consistent with previous results examining interactive effects between childhood trauma and the 5-HTTLPR genotype (Xie, Kranzler, Farrer, \& Gelernter, 2012). The results of the current study are unique in testing interactive effects with both childhood and combat trauma and utilizing a latent variable model of PTSD in a sample of veterans. 


\subsection{Indirect associations between PTSD symptoms and externalizing problems}

Consistent with previous research, affect lability exhibited direct associations with alcohol dependence syndrome symptoms over and above the effects of alcohol consumption (Simons et al., 2009; Simons, Oliver, et al., 2005b; Simons et al., 2014) and disinhibition exhibited direct associations with conduct related problems (Simons et al., 2009). Previous research has indicated that negative affectivity and disinhibition mediate associations between PTSD symptoms and substance use problems (Miller et al., 2006). The current study extends previous findings to show that these symptoms of emotional and behavioral dysregulation may underlie varying profiles of externalizing problems in veterans. Consistent with the negative reinforcement model of substance dependence, we posit that affective instability confers unique risk to the development of compulsive drinking patterns (Baker et al., 2004; McCarthy et al., 2010).

Veterans with PTSD exhibit a wide range of a conduct related problems including interpersonal violence, impairments in social relationships, and broad difficulties in sociooccupational functioning (Davis et al., 2012; Jakupcak et al., 2007; Meis, Erbes, Polusny, \& Compton, 2010; Schnurr, Lunney, Bovin, \& Marx, 2009). The results of the current study highlight the role of behavioral disinhibition mediating effects of PTSD symptoms on associated conduct related problems. PTSD symptoms may contribute to impulse control problems, which contribute to problems in adaptive functioning. The chronic stress associated with managing symptoms may reduce self-control resources resulting in inability to maintain control of behavior consistent with long-term goals (Friese et al., 2009).

PTSD symptoms were indirectly associated with the outcomes via increased alcohol consumption. However, the associations via affect lability and behavioral disinhibition are consistent with the premise that associations between PTSD symptoms and alcohol-related problems reflect more than a self-medication type process, which would be accounted for by increases in consumption. Rather alcohol-related problems among veterans may reflect broader deficits in self-regulation stemming from PTSD (Gaher et al., 2014).

\section{Limitations}

Several limitations should be noted. First, the cross-section design precludes causal inferences. Although the structural model fit well, alternative models cannot be ruled out. For example, temperament may be a preexisting risk factor for PTSD (Gaher et al., 2014; Meis et al., 2010). Second, the sample size was modest for the complexity of the latent variable model and for testing the $\mathrm{G} \times \mathrm{E}$ interactions. Third, observed genetic effects are conditional upon context, both genetic and environmental. Additional research with larger samples, longitudinal designs, and testing of additional moderators is warranted to further test the hypotheses.

\section{Summary}

The results indicate that deficits in behavioral and emotional regulation may be interme-

diate traits underlying conduct related problems and symptoms of alcohol dependence 
syndrome, respectively. Increased risk of externalizing problems associated with PTSD reflect deficits in emotional and behavioral control. Childhood traumatic stress confers increased risk for PTSD symptoms among veterans with the low functioning alleles of the 5-HTTLPR polymorphism.

Acknowledgments - This research was supported in part by the National Institute on Alcohol Abuse and Alcoholism of the National Institutes of Health under Award Number R01AA020519 (to Jeffrey S. Simons). The content is solely the responsibility of the authors and does not necessarily represent the official views of the National Institutes of Health, the Department of Veterans Affairs, or the United States Government. This research was supported with resources and the use of facilities at the Sioux Falls VA Medical Center, Bay Pines VA Health Care System, and the University of South Dakota.

Contributors - All authors contributed to and have approved the final manuscript.

Conflict of interest - All authors declare they have no conflicts of interest.

\section{Note}

1. We thank an anonymous reviewer for suggesting that quadratic effects of the traumatic stress variables should be tested for the purpose of ruling out spurious interactions. We did this as requested. Neither the combat trauma quadratic term $(\beta=-0.14$ (SE $=0.07), p=0.057)$ nor the childhood trauma quadratic effect $(\beta=-0.07$ ( $\mathrm{SE}=0.09), p=0.419)$ were significant and the $\mathrm{G} \times \mathrm{E}$ interaction remained significant while controlling for them. The reviewer also suggested that the stress inoculation hypothesis may reflect a quadratic effect of childhood stress interacting with combat trauma. Hence, we also tested this interaction (including the requisite lower order terms). The effect was not significant $(\beta=0.11(\mathrm{SE}=0.10), p=0.268)$. In each permutation, the $\mathrm{G} \times \mathrm{E}$ interaction remained significant lending further confidence in the result.

\section{References}

Aiken, L. S., \& West, S. G. (1991). Multiple regression: Testing and interpreting interactions. London: Sage.

Baker, T. B., Piper, M. E., McCarthy, D. E., Majeskie, M. R., \& Fiore, M. C. (2004). Addiction motivation reformulated: An affective processing model of negative reinforcement. Psychological Review, 111, 33-51. http://dx.doi.org/10.1037/0033-295X.111.1.33

Blanchard, E. B., Jones-Alexander, J., Buckley, T. C., \& Forneris, C. A. (1996). Psychometric properties of the PTSD checklist (PCL). Behaviour Research and Therapy, 34, 669-673.

Carver, C. S. (2005). Impulse and constraint: Perspectives from personality psychology, convergence with theory in other areas, and potential for integration. Personality and Social Psychology Review, 9, 312-333.

Davis, L. L., Leon, A. C., Toscano, R., Drebing, C. E., Ward, L. C., Parker, P. E., . . Drake, R. E. (2012). A randomized controlled trial of supported employment among veterans with posttraumatic stress disorder. Psychiatric Services, 63, 464-470.

Dimeff, L. A., Baer, J. S., Kivlahan, D. R., \& Marlatt, G. A. (1999). Brief alcohol screening and intervention for college students (BASICS): A harm reduction approach. New York: Guilford Press. 
Dougherty, L. R., Klein, D. N., \& Davila, J. (2004). A growth curve analysis of the course of dysthymic disorder: The effects of chronic stress and moderation by adverse parent-child relationships and family history. Journal of Consulting and Clinical Psychology, 72, 1012.

Doyle, S. R., \& Donovan, D. M. (2009). A validation study of the alcohol dependence scale. Journal of Studies on Alcohol and Drugs, 70, 689-699.

Driessen, M., Schulte, S., Luedecke, C., Schaefer, I., Sutmann, F., Ohlmeier, M., . . Group, T. R.-S. (2008). Trauma and PTSD in patients with alcohol, drug, or dual dependence: A multi-center study. Alcoholism, Clinical and Experimental Research, 32, 481-488. http://dx.doi.org/10.1111/ j.1530-0277.2007.00591.x

Ducci, F., \& Goldman, D. (2008). Genetic approaches to addiction: Genes and alcohol. Addiction, 103, 1414-1428. http://dx.doi.org/10.1111/j.1360-0443.2008.02203.x

Eysenck, S. B., Pearson, P. R., Easting, G., \& Allsopp, J. F. (1985). Age norms for impulsiveness, venturesomeness and empathy in adults. Personality and Individual Differences, 6, 613-619.

Fergus, S., \& Zimmerman, M. A. (2005). Adolescent resilience: A framework for understanding healthy development in the face of risk. Annual Review of Public Health, 26, 399-419.

Fernie, G., Peeters, M., Gullo, M. J., Christiansen, P., Cole, J. C., Sumnall, H., \& Field, M. (2013). Multiple behavioural impulsivity tasks predict prospective alcohol involvement in adolescents. Addiction, 108, 1916-1923. http://dx.doi.org/10.1111/add. 12283

Friese, M., Wanke, M., Hofmann, W., Forgas, J. P., Baumeister, R. F., \& Tice, D. M. (2009). Unscrambling self-regulatory behavior determination: The interplay of impulse strength, reflective processes, and control resources. Psychology of self-regulation: Cognitive, affective, and motivational processes (pp. 53-71). New York, NY US: Psychology Press.

Fulton, J. J., Calhoun, P. S., Wagner, H. R., Schry, A. R., Hair, L. P., Feeling, N., . . Beckham, J. C. (2015). The prevalence of posttraumatic stress disorder in operation enduring freedom/operation Iraqi freedom (OEF/OIF) veterans: A meta-analysis. Journal of Anxiety Disorders, 31, 98-107. http://dx.doi.org/10.1016/j.janxdis.2015.02.003

Gaher, R. M., Simons, J. S., Hahn, A. M., Hofman, N. L., Hansen, J., \& Buchkoski, J. (2014). An experience sampling study of PTSD and alcohol-related problems. Psychology of Addictive Behaviors, 28, 1013-1025. http://dx.doi.org/10.1037/a0037257

Garmezy, N. (1991). Resilience in children's adaptation to negative life events and stressed environments. Pediatric Annals, 20(459-460), 463-456.

Giancola, P. R., Parrott, D. J., \& Roth, R. M. (2006). The influence of difficult temperament on alcoholrelated aggression: Better accounted for by executive functioning? Addictive Behaviors, 31, 21692187.

Gillespie, C. F., Phifer, J., Bradley, B., \& Ressler, K. J. (2009). Risk and resilience: Genetic and environmental influences on development of the stress response. Depression and Anxiety, 26, 984-992.

Gressier, F., Calati, R., Balestri, M., Marsano, A., Alberti, S., Antypa, N., \& Serretti, A. (2013). The 5-HTTLPR polymorphism and posttraumatic stress disorder: A meta-analysis. Journal of Traumatic Stress, 26, 645-653. http://dx.doi.org/10.1002/jts.21855

Harkness, K. L., Bruce, A. E., \& Lumley, M. N. (2006). The role of childhood abuse and neglect in the sensitization to stressful life events in adolescent depression. Journal of Abnormal Psychology, 115,730 .

Hicks, B. M., Blonigen, D. M., Kramer, M. D., Krueger, R. F., Patrick, C. J., Iacono, W. G., \& McGue, M. (2007). Gender differences and developmental change in externalizing disorders from late adolescence to early adulthood: A longitudinal twin study. Journal of Abnormal Psychology, 116, 433-447. http://dx.doi.org/10.1037/0021-843X. 116.3.433 
Hoge, C. W., Castro, C. A., Messer, S. C., McGurk, D., Cotting, D. I., \& Koffman, R. L. (2004). Combat duty in Iraq and Afghanistan, mental health problems, and barriers to care. New England Journal of Medicine, 351, 13-22.

Hoge, C. W., Auchterlonie, J. L., \& Milliken, C. S. (2006). Mental health problems, use of mental health services, and attrition from military service after returning from deployment to Iraq or Afghanistan. JAMA: The Journal of the American Medical Association, 295, 1023-1032.

Jacobson, I. G., Donoho, C. J., Crum-Cianflone, N. F., \& Maguen, S. (2015). Longitudinal assessment of gender differences in the development of PTSD among US military personnel deployed in support of the operations in Iraq and Afghanistan. Journal of Psychiatric Research, 68, 30-36. http://dx.doi.org/10.1016/j.jpsychires.2015. 05.015

Jakupcak, M., Conybeare, D., Phelps, L., Hunt, S., Holmes, H. A., Felker, B., . . McFall, M. E. (2007). Anger, hostility, and aggression among Iraq and Afghanistan war veterans reporting PTSD and subthreshold PTSD. Journal of Traumatic Stress, 20, 945-954.

Kendall, P. C., \&Williams, C. L. (1982). Assessing the cognitive and behavioral components of children's self-management. In P. Karoly, \& F. H. Kanfer (Eds.), Self-management and behavior change (pp. 240-284). New York: Pergamon Press.

Kendler, K. S., Kuhn, J. W., \& Prescott, C. A. (2004). Childhood sexual abuse, stressful life events and risk for major depression in women. Psychological Medicine, 34, 1475-1482.

Khoshaba, D. M., \& Maddi, S. R. (1999). Early experiences in hardiness development. Consulting Psychology Journal: Practice and Research, 51, 106.

Kimbrel, N. A., Morissette, S. B., Meyer, E. C., Chrestman, R., Jamroz, R., Silvia, P. J., . . Young, K. A. (2015). Effect of the 5-HTTLPR polymorphism on posttraumatic stress disorder, depression, anxiety, and quality of life among Iraq and Afghanistan veterans. Anxiety, Stress, and Coping, 28, 456-466. http://dx.doi.org/10.1080/10615806.2014.973862

Koenen, K. C., Nugent, N. R., \& Amstadter, A. B. (2008). Gene-environment interaction in posttraumatic stress disorder: Review, strategy and new directions for future research. European Archives of Psychiatry and Clinical Neuroscience, 258, 82-96. http://dx.doi.org/10.1007/s00406-0070787-2

Liu, Y., Garrett, M. E., Dennis, M. F., Green, K. T., Workgroup, V. A.M.-A.M. R., Ashley-Koch, A. E., \& Kimbrel, N. A. (2015). An examination of the association between 5-HTTLPR, combat exposure, and PTSD diagnosis among U.S. veterans. PLoS ONE, 10, e0119998. http://dx.doi.org/ 10.1371/journal.pone.0119998

Lyons, D. M., \& Parker, K. J. (2007). Stress inoculation-induced indications of resilience in monkeys. Journal of Traumatic Stress, 20, 423-433.

Masten, A. S. (2001). Ordinary magic: Resilience processes in development. American Psychologist, $56,227$.

McCarthy, D. E., Curtin, J. J., Piper, M. E., \& Baker, T. B. (2010). Negative reinforcement: Possible clinical implications of an integrative model. In J. D. Kassel (Ed.), Substance abuse and emotion (pp. 15-42). Washington, DC US: American Psychological Association.

MacKinnon, D. P., Lockwood, C. M., \&Williams, J. (2004). Confidence limits for the indirect effect: Distribution of the product and resampling methods. Multivariate Behavioral Research, 39(1), 99-128.

Meaney, M. J., \& Szyf, M. (2005). Environmental programming of stress responses through DNA methylation: Life at the interface between a dynamic environment and a fixed genome. Dialogues in Clinical Neuroscience, 7, 103. 
Meis, L. A., Erbes, C. R., Polusny, M. A., \& Compton, J. S. (2010). Intimate relationships among returning soldiers: The mediating and moderating roles of negative emotionality, PTSD symptoms, and alcohol problems. Journal of Traumatic Stress, 23, 564-572.

Miller, W., Tonigan, J., \& Longabaugh, R. (1995). The drinker inventory of consequences (DrINC): An instrument for assessing adverse consequences of alcohol abuse. Test manual (Vol. 4, project MATCH monograph series). Rockville, MD: National Institute on Alcohol Abuse and Alcoholism.

Miller, M. W., Vogt, D. S., Mozley, S. L., Kaloupek, D. G., \& Keane, T. M. (2006). PTSD and substancerelated problems: The mediating roles of disconstraint and negative emotionality. Journal of Abnormal Psychology, 115, 369-379.

Mortimer, J. T., \& Staff, J. (2004). Early work as a source of developmental discontinuity during the transition to adulthood. Development and Psychopathology, 16, 1047-1070.

Muthén, L. K., \& Muthén, B. O. (2015). Mplus Statistical Modeling Software: Release 7.4. Los Angeles, CA: Muthén \& Muthén.

Nakamura, M., Ueno, S., Sano, A., \& Tanabe, H. (2000). The human serotonin transporter gene linked polymorphism (5-HTTLPR) shows ten novel allelic variants. Molecular Psychiatry, 5, 32-38.

Navarro-Mateu, F., Escamez, T., Koenen, K. C., Alonso, J., \& Sanchez-Meca, J. (2013). Meta-analyses of the 5-HTTLPR polymorphisms and post-traumatic stress disorder. PloS One, 8, e66227. http://dx.doi.org/10.1371/journal.pone.0066227.

Neal, D. J., \& Carey, K. B. (2007). Association between alcohol intoxication and alcohol-related problems: An event-level analysis. Psychology of Addictive Behaviors, 21, 194-204.

Oliver, M. N. I., \& Simons, J. S. (2004). The affective lability scales: Development of a short-form measure. Personality and Individual Differences, 37, 1279-1288. http://dx.doi.org/10.1016/j.paid .2003 .12 .013

Ouimette, P. C., Brown, P. J., \& Najavits, L. M. (1998). Course and treatment of patients with both substance use and posttraumatic stress disorders. Addictive Behaviors, 23, 785-795.

Pietrzak, R. H., Goldstein, R. B., Southwick, S. M., \& Grant, B. F. (2011). Prevalence and Axis I comorbidity of full and partial posttraumatic stress disorder in the United States: Results from wave 2 of the national epidemiologic survey on alcohol and related conditions. Journal of Anxiety Disorders, 25, 456-465. http://dx.doi.org/10.1016/j.janxdis. 2010.11.010

Pietrzak, R. H., Galea, S., Southwick, S. M., \& Gelernter, J. (2013). Examining the relation between the serotonin transporter 5-HTTPLR genotype $\times$ trauma exposure interaction on a contemporary phenotypic model of posttraumatic stress symptomatology: A pilot study. Journal of Affective Disorders, 148, 123-128. http://dx.doi.org/10.1016/j. jad.2012.11.003

Samuel, D. B., South, S. C., \& Griffin, S. A. (2015). Factorial invariance of the Five-Factor model rating form across gender. Assessment, 22, 65-75. http://dx.doi.org/10.1177/ 1073191114536772

Scherrer, J. F., Xian, H., Lyons, M. J., Goldberg, J., Eisen, S. A., True, W. R., . . Koenen, K. C. (2008). Posttraumatic stress disorder; combat exposure; and nicotine dependence, alcohol dependence, and major depression in male twins. Comprehensive Psychiatry, 49, 297-304. http://dx.doi.org/10 .1016/j.comppsych.2007.11.001.

Schnurr, P. P., Lunney, C. A., Bovin, M. J., \&Marx, B. P. (2009). Posttraumatic stress disorder and quality of life: Extension of findings to veterans of the wars in Iraq and Afghanistan. Clinical Psychology Review, 29, 727-735.

Seal, K. H., Metzler, T. J., Gima, K. S., Bertenthal, D., Maguen, S., \& Marmar, C. R. (2009). Trends and risk factors for mental health diagnoses among Iraq and Afghanistan veterans using department 
of veterans affairs health care, 2002-2008. American Journal of Public Health, 99, 1651-1658. http://dx.doi.org/10.2105/AJPH.2008.150284

Seal, K. H., Cohen, G., Waldrop, A., Cohen, B. E., Maguen, S., \& Ren, L. (2011). Substance use disorders in Iraq and Afghanistan veterans in VA healthcare, 2001-2010: Implications for screening, diagnosis and treatment. Drug and Alcohol Dependence, 116, 93-101.

Shapero, B. G., Black, S. K., Liu, R. T., Klugman, J., Bender, R. E., Abramson, L. Y., \& Alloy, L. B. (2014). Stressful life events and depression symptoms: The effect of childhood emotional abuse on stress reactivity. Journal of Clinical Psychology, 70, 209-223.

Sher, K. J., \& Trull, T. J. (1994). Personality and disinhibitory psychopathology: Alcoholism and antisocial personality disorder. Journal of Abnormal Psychology, 103, 92-102.

Sher, K. J., Dick, D. M., Crabbe, J. C., Hutchison, K. E., O'Malley, S. S., \& Heath, A. C. (2010). Consilient research approaches in studying gene $\times$ environment interactions in alcohol research. Addiction Biology, 15, 200-216. http://dx.doi.org/10.1111/j.1369-1600. 2009.00189.x

Simons, J. S., Gaher, R. M., Oliver, M. N. I., Bush, J. A., \& Palmer, M. A. (2005a). An experience sampling study of associations between affect and alcohol use and problems among college students. Journal of Studies on Alcohol, 66, 459-469.

Simons, J. S., Oliver, M. N., Gaher, R. M., Ebel, G., \& Brummels, P. (2005b). Methamphetamine and alcohol abuse and dependence symptoms: Associations with affect lability and impulsivity in a rural treatment population. Addictive Behaviors, 30, 1370-1381.

Simons, J. S., Oliver, M. N. I., Gaher, R. M., Ebel, G., \& Brummels, P. (2005c). Methamphetamine and alcohol abuse and dependence symptoms: Associations with affect lability and impulsivity in a rural treatment population. Addictive Behaviors, 30, 1370-1381. http://dx.doi.org/10.1016/j.addbeh .2005 .01 .018

Simons, J. S., Carey, K. B., \&Wills, T. A. (2009). Alcohol abuse and dependence symptoms: A multidimensional model of common and specific etiology. Psychology of Addictive Behaviors, 23, 415-427. http://dx.doi.org/10.1037/a0016003

Simons, J. S., Wills, T. A., \& Neal, D. J. (2014). The many faces of affect: A multilevel model of drinking frequency/quantity and alcohol dependence symptoms among young adults. Journal of Abnormal Psychology, 123, 676-694. http://dx.doi.org/10.1037/a0036926

Simons, J. S., Wills, T. A., Emery, N. N., \& Spelman, P. J. (2015). Keep calm and carry on: Maintaining self-control when intoxicated, upset, or depleted. Cognition and Emotion, 1-15. http://dx.doi.org/ 10.1080/02699931.2015.1069733

Skinner, H. A., \& Allen, B. A. (1982). Alcohol dependence syndrome: Measurement and validation. Journal of Abnormal Psychology, 91, 199-209.

Skinner, H. A., \& Horn, J. L. (1984). Alcohol dependence scale: User's guide. Toronto: Addiction Research Foundation.

Tarter, R. E. (2002). Etiology of adolescent substance abuse: A developmental perspective. The American Journal on Addictions, 11, 171-191.

Tate, S. R., Norman, S. B., McQuaid, J. R., \& Brown, S. A. (2007). Health problems of substance-dependent veterans with and those without trauma history. Journal of Substance Abuse Treatment, 33, 25-32.

U.S. Department of Veteran Affairs (2012). Using the PTSD checklist (PCL). (Retrieved from) http:// www.ptsd.va.gov/professional/pages/assessments/ptsd-checklist.asp

Vogt, D., Smith, B., King, D., \& King, L. (2012). Manual for the deployment risk and resilience inventory-2 (DRRI-2): A collection of measures for studying deployment-related experiences of military veterans. Boston, MA: National Center for PTSD. 
Wald, I., Degnan, K. A., Gorodetsky, E., Charney, D. S., Fox, N. A., Fruchter, E., . . Bar-Haim, Y. (2013). Attention to threats and combat-related posttraumatic stress symptoms: Prospective associations and moderation by the serotonin transporter gene. JAMA Psychiatry, 70, 401-408. http://dx.doi.org/10.1001/2013.jamapsychiatry.188.

Walsh, K., Uddin, M., Soliven, R., Wildman, D. E., \& Bradley, B. (2014). Associations between the SS variant of 5-HTTLPR and PTSD among adults with histories of childhood emotional abuse: Results from two African American independent samples. Journal of Affective Disorders, 161, 9196. http://dx.doi.org/10.1016/j.jad.2014.02.043

Wang, Z., Baker, D. G., Harrer, J., Hamner, M., Price, M., \& Amstadter, A. (2011). The relationship between combat-related posttraumatic stress disorder and the 5-HTTLPR/rs25531 polymorphism. Depression and Anxiety, 28, 1067-1073. http://dx.doi.org/10.1002/da.20872

Weathers, F., Litz, B., Herman, D., Huska, J., \& Keane, T. (1993). The PTSD checklist (PCL): Reliability, validity, and diagnostic utility. San Antonio, TX: Paper presented at the International Society of Traumatic Stress Studies. http://www.pdhealth.mil/library/downloads/PCL_sychometrics.doc

Wendland, J. R., Martin, B. J., Kruse, M. R., Lesch, K. P., \& Murphy, D. L. (2006). Simultaneous genotyping of four functional loci of human SLC6A4, with a reappraisal of 5-HTTLPR and rs25531. Molecular Psychiatry, 11, 224-226. http://dx.doi.org/10.1038/sj.mp.4001789

Wills, T. A., Pokhrel, P., Morehouse, E., \& Fenster, B. (2011). Behavioral and emotional regulation and adolescent substance use problems: A test of moderation effects in a dual-process model. Psychology of Addictive Behaviors, 25, 279-292. http://dx.doi. org/10.1037/a0022870

Wills, T. A., Bantum, E. O., Pokhrel, P., Maddock, J. E., Ainette, M. G., Morehouse, E., \& Fenster, B. (2013). A dual-process model of early substance use: Tests in two diverse populations of adolescents. Health Psychology, 32, 533-542. http://dx.doi.org/10.1037/a0027634

Xie, P., Kranzler, H. R., Farrer, L., \& Gelernter, J. (2012). Serotonin transporter 5-HTTLPR genotype moderates the effects of childhood adversity on posttraumatic stress disorder risk: A replication study. American Journal of Medical Genetics. Part B, Neuropsychiatric Genetics, 159B, 644-652. http://dx.doi.org/10.1002/ajmg.b.32068 